Article

\title{
Synthesis, Cytotoxic Activity and 2D-QSAR Study of Some Imidazoquinazoline Derivatives
}

\author{
Hanan Georgey \\ Department of Pharmaceutical Chemistry, Faculty of Pharmacy, Cairo University, \\ Cairo P.O. Box 11562, Egypt; E-Mail: hanan-hanna@hotmail.com; Tel.: +2-02-253-531-00; \\ Fax: +2-02-253-200-05
}

Received: 14 January 2014; in revised form: 17 March 2014 / Accepted: 19 March 2014 / Published: 24 March 2014

\begin{abstract}
A novel series of 4-substituted amino-7,8-dimethoxy-1-phenylimidazo[1,5a] quinazolin-5(4H)-one derivatives was designed, synthesized and tested for their antitumor activity against a human mammary carcinoma cell line (MCF7). Compound 5a was found to be the most active derivative. Physico-chemical parameters were also determined and revealed that most of the compounds obeyed the "rule of five" properties with good absorption percentages. 2D-QSAR studies revealed a well predictive and statistically significant and cross validated QSAR model that helps to explore some expectedly potent compounds.
\end{abstract}

Keywords: imidazolinone; imidazo[1,5-a]quinazoline; antitumor activity; Lipinski’s parameters; 2D-QSAR

\section{Introduction}

Struggling efforts for the treatment and eradication of cancer have grown tremendously in the last few years led to the decrease in cancer death rates by $1.8 \%$ per year in men and by $1.5 \%$ per year in women [1]. Cancer is characterized by a deregulation of the cell cycle, resulting in progressive loss of the cellular differentiation and non-controlled cellular growth $[2,3]$. The control of disseminated tumor growth by systemically active chemotherapeutic agents remains a major challenge for cancer chemotherapy, despite decades of focused efforts. Although there are some notable successes with certain forms of cancer [4], the discovery and development of novel therapeutic agents for the treatment of cancer has a vital importance [5-11].

Imidazole, a small bioactive molecule, is a prominent structural motif found in numerous biologically active compounds. Interestingly, imidazolinones such as I [12] (Figure 1) and methoxyquinazoline 
derivatives II, geftinib III and IV [13-16] (Figure 1) all display significant antitumor activity. Hybrid molecules combining the imidazole and quinazoline moieties in either linear or angular imidazoquinazolines were designed and demonstrated promising antitumor activity [17-19]. Furthermore, incorporation of other heterocyclic moieties such as piperazine [20,21], piperidine [22,23], imidazole [24] and triazine [25] moieties was reported to enhance the antitumor activity too.

Figure 1. Structures of some active antitumor agents from the literature.

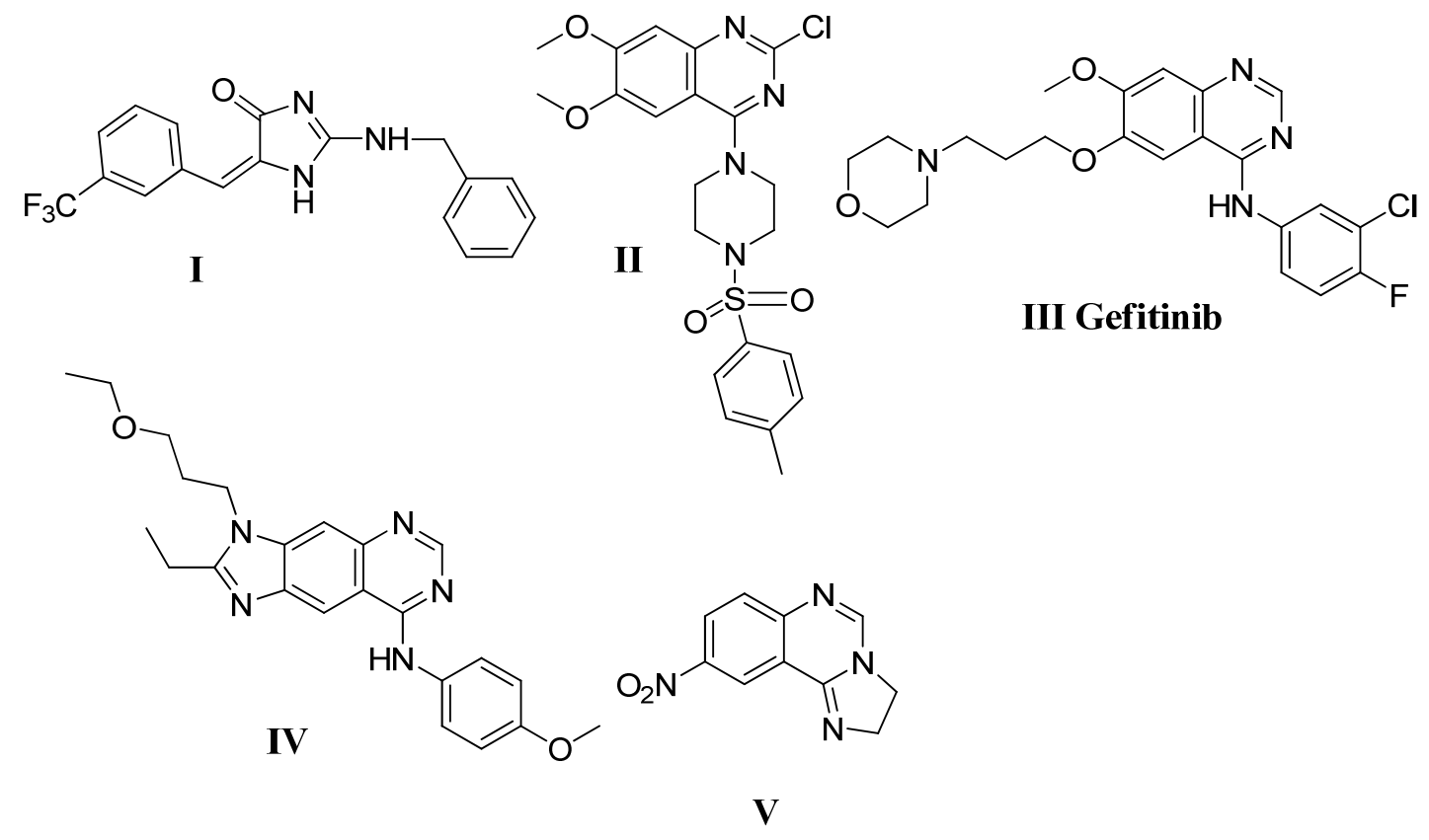

Inspired by the aforementioned facts, it was deemed of interest to use the hybridized 7,8-dimethoxyimidazoquinazoline ring system as a scaffold for the design of new molecules with potential antitumor activity. The target compounds incorporate different heterocyclic moieties such as piperazine, piperidine, or imidazole. The novel compounds were tested for their antitumor activity. Physico-chemical parameters were also calculated to obtain their "rule of five" properties. Moreover, 2D-QSAR studies were also applied to correlate between the structures of the synthesized compounds and their pharmacological activities.

\section{Results and Discussion}

\subsection{Chemistry}

The synthetic strategy for the preparation of the target compounds $\mathbf{2 a}, \mathbf{b}, \mathbf{3 a}, \mathbf{b}, \mathbf{4}, \mathbf{5 a}, \mathbf{b}, \mathbf{6 a}-\mathbf{d}, \mathbf{7 a}-\mathbf{d}$, $\mathbf{8}$ and $\mathbf{9}$ is illustrated in Schemes 1-3. Imidazolinone derivatives 2a,b were obtained through the reaction of oxazolone derivatives 1a,b, previously synthesized according to the literature method [26,27] with methyl 2-amino-4,5-dimethoxybenzoate in glacial acetic acid in the presence of sodium acetate. This reaction proceeded through open intermediates which were recyclized in the presence of glacial acetic acid to afford the imidazolinones [28]. Reaction of $\mathbf{2 a}, \mathbf{b}$ with hydrazine hydrate afforded either the benzohydrazide derivatives $\mathbf{3 a}, \mathbf{b}$ or imidazo[1,5-a]quinazolinone derivative $\mathbf{4}$ depending on the reaction conditions. 
Scheme 1. Synthesis of the target compounds 1-4.<smiles>[R7]c1ccc(/C=C2\N=C(c3ccccc3)OC2=O)cc1</smiles>

1a; $\mathrm{R}_{1}=\mathrm{H}$

1b; $\mathrm{R}_{1}=\mathrm{OCH}_{3}$

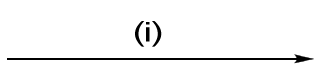

(ii)<smiles>[R16]c1ccc(/C=C2\N=C(c3ccccc3)N(c3cc(OC)c(OC)cc3C(=O)NN)C2=O)cc1</smiles>

3a; $\mathrm{R}_{1}=\mathrm{H}$

3b; $\mathrm{R}_{1}=\mathrm{OCH}_{3}$<smiles>[R10][CH][R7]</smiles>

$\mathbf{2 b} ; \mathrm{R}_{1}=\mathrm{OCH}_{3}$

(iii)

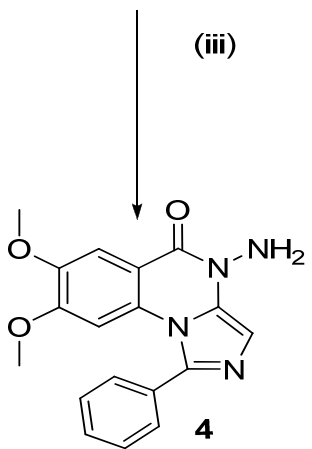

Reagents and conditions: (i) methyl 2-amino-4,5-dimethoxybenzoate, $\mathrm{CH}_{3} \mathrm{COOH}, \mathrm{CH}_{3} \mathrm{COONa}$; (ii) Hydrazine hydrate, methanol, 2 h; (iii) Hydrazine hydrate, $80 \%$ ethanol, 24 h.

Scheme 2. Synthesis of the target compounds 5-8.<smiles>C/C=C\CC</smiles>

8 (i)<smiles>COc1cc2c(=O)n(NC(=O)CCl)c3cnc(-c4ccccc4)n3c2cc1OC</smiles>

(iii)

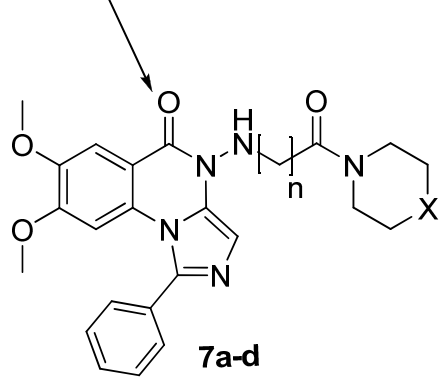

(ii)
$5 \mathbf{a} ; n=1$
$\mathbf{5 b} ; n=2$

$$
\begin{aligned}
& \text { 6a, 7a; } \mathrm{n}=1, \mathrm{X}=\mathrm{CH}_{2} \\
& \text { 6b, 7b; } n=1, X=\mathrm{N}-\mathrm{CH}_{3} \\
& 6 c, 7 c ; n=2, X=\mathrm{CH}_{2} \\
& \text { 6d, 7d; } n=2, X=N-\mathrm{CH}_{3}
\end{aligned}
$$

Reagents and conditions: (i) $\mathrm{Cl}\left(\mathrm{CH}_{2}\right)_{\mathrm{n}} \mathrm{COCl}$, dry DMF; (ii) Piperidine or N-methylpiperazine, acetonitrile, $\mathrm{K}_{2} \mathrm{CO}_{3}$; (iii) 2/3-chloro-1-(piperidin-1-yl/4-methylpiperazin-1-yl)ethanone/propan-1-one, dry DMF, triethylamine; (iv) 2-chloroacetamide, dry DMF. 
Scheme 3. Synthesis of the target compound 9.<smiles>COc1cc2c(=O)n(NC(=O)CCl)c3cnc(-c4ccccc4)n3c2cc1OC</smiles>

$5 a$ (i)

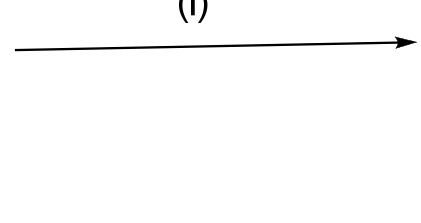

Reagents and conditions: (i) KCNO, acetic acid.

Refluxing 2a,b with equimolar amounts of hydrazine hydrate in methanol for $2 \mathrm{~h}$ yielded the benzohydrazide derivatives 3a,b, whereas, the imidazoquinazoline derivative $\mathbf{4}$ was obtained according to the reported reaction conditions [29] using excess hydrazine hydrate in aqueous ethanol $80 \%$ at reflux for $24 \mathrm{~h}$. (Scheme 1). On the other hand, the reaction of compound 4 with chloroacetyl chloride in DMF led to the formation of 2-chloro- $N$-(7,8-dimethoxy-5-oxo-1-phenylimidazo[1,5-a]quinazolin$5(4 H)$-yl)acetamide (5a). Similarly, the reaction with chloropropionyl chloride gave $\mathbf{5 b}$. Reaction of $\mathbf{5 a}, \mathbf{b}$ with secondary amines (namely, piperidine and $N$-methylpiperazine) in dry acetonitrile in the presence of potassium carbonate yielded compounds $\mathbf{6 a}-\mathbf{d}$. Ethylamino or propylamino-1phenylimidazo[1,5-a]quinazolin-5(4H)-one derivatives 7a-d were obtained by reacting compound 4 with 2/3-chloro-1-(piperidin-1-yl/4-methylpiperazin-1-yl)ethanone/propan-1-one in dry DMF in the presence of triethylamine. Moreover, formation of the oxotriazine ring was achieved via the reaction of the $N$-amino derivative 4 with chloroacetamide in DMF to yield 11,12-dimethoxy-8-phenyl-3,4dihydro-2 $H$-imidazo[1,5-a][1,2,4]triazino[2,3-c] quinazolin-2-one (8) as depicted in Scheme 2. As shown in Scheme 3, the cyclized product 9 was obtained starting with the chloroacetamide derivative $\mathbf{5 a}$. Reaction with potassium cyanate yielded the 1-(7,8-dimethoxy-5-oxo-1-phenylimidazo[1,5a]quinazolin-4(5H)-yl)-1H-imidazole-2,5-dione(9). The newly synthesized compounds were confirmed via elemental analyses and spectral data (IR, ${ }^{1} \mathrm{H}-\mathrm{NMR},{ }^{13} \mathrm{C}-\mathrm{NMR}$ and mass spectra).

\subsection{Preliminary in-Vitro Antitumor Screening}

All the synthesized compounds were tested for their antitumor activity against human mammary carcinoma cell line (MCF7) at the National Cancer Institute, Cairo University [30]. From the observed antitumor data (Table 1), it was noticed that imidazolinone derivative 3a and imidazoquinazoline derivatives 5a, 6a, 7a and 7b showed $\mathrm{IC}_{50}$ values of 27.1, 10.6, 26.4, 21.6 and $26.8 \mu \mathrm{M}$, respectively. Also, compounds 5a and $7 \mathbf{a}$ revealed remarkable activity comparable to that of the reference drug mitoxantrone.

Structure-activity correlations, based on the human mammary carcinoma cell line (MCF7), revealed that the chloroacetyl derivative 5a was more active than its chloropropionyl analogue $\mathbf{5 b}$. Regarding the effect of the substituent on $\mathbf{5 a}, \mathbf{b}$, the results of $\mathbf{6 a - d}$ and $7 \mathbf{a}-\mathbf{d}$ showed that a piperidinyl moiety was more active than a $N$-methylpiperazinyl one, and an acetyl moiety was more active than its corresponding propionyl moiety. Also, derivatives in which the carbonyl moiety was directly attached 
to a piperidinyl/piperazinyl (compounds $\mathbf{7 a - d}$ ) were more active than those in which the carbonyl was attached to an $N$-aminoimidazoquinazoline (compounds $\mathbf{6 a - d}$ ).

Table 1. Cytotoxic activity of the newly synthesized compounds against breast cancer MCF7 cell line.

\begin{tabular}{ccc}
\hline Compd. No & IC $_{\mathbf{5 0}} \boldsymbol{\mu g} / \mathbf{m L}$ & $\mathbf{I C}_{\mathbf{5 0}} \boldsymbol{\mu M}$ \\
\hline $\mathbf{2 a}$ & 18.0 & 40.6 \\
$\mathbf{2 b}$ & 21.8 & 46.1 \\
$\mathbf{3 a}$ & 12.0 & 27.1 \\
$\mathbf{3 b}$ & 42.4 & 89.7 \\
$\mathbf{4}$ & 12.2 & 36.2 \\
$\mathbf{5 a}$ & 4.4 & 10.6 \\
$\mathbf{5 b}$ & 14.0 & 32.8 \\
$\mathbf{6 a}$ & 12.2 & 26.4 \\
$\mathbf{6 b}$ & 17.2 & 36.0 \\
$\mathbf{6 c}$ & 19.0 & 39.9 \\
$\mathbf{6 d}$ & 21.4 & 43.6 \\
$\mathbf{7 a}$ & 10.0 & 21.6 \\
$\mathbf{7 b}$ & 12.8 & 26.8 \\
$\mathbf{7 c}$ & 17.4 & 36.5 \\
$\mathbf{7 d}$ & 18.4 & 37.5 \\
$\mathbf{8}$ & 21 & 56.1 \\
$\mathbf{9}$ & 19.0 & 45.5 \\
Mitoxantrone & 10.1 & 22.7 \\
\hline
\end{tabular}

\subsection{Physico-Chemical Parameters}

Computational study for the prediction of physico-chemical parameters of all synthesized compounds has been executed. The computed molecular properties are shown in Table 2. Numbers of rotatable bonds, $\log P$, molecular polar surface area (PSA), number of hydrogen bond accepter and donor atoms of Lipinski's "rule of five" [31] were calculated using the Molinspiration online property calculation toolkit [32]. The degree of absorption is expressed by the percentage of absorption $(\% \mathrm{ABS})$, that was calculated by using $\% \mathrm{ABS}=109-(0.345 \times \mathrm{TPSA})$ [33]. From all these parameters, it was found that, all the synthesized derivatives exhibited \%ABS ranging from $61.41 \%$ to $84.91 \%$. Furthermore, most of the compounds obeyed the "rule of five" properties.

\subsection{D-QSAR Studies}

A 2D-QSAR study was performed in order to find a mathematical correlation between the structures of the newly synthesized compounds and their antitumor activity [34] that expressed as $-\log \mathrm{IC}_{50}$ values, using Molecular Operating Environment (MOE) software package. 
Table 2. Calculated absorption (\%ABS), polar surface area (PSA) and Lipinski parameters of the newly synthesized compounds.

\begin{tabular}{ccccccccc}
\hline Compd. No & \%ABS & TPSA & nrotb & nON $\leq \mathbf{1 0}$ & nOHNH $\leq \mathbf{5}$ & miLogP $\leq \mathbf{5}$ & MW $\leq \mathbf{5 0 0}$ & n violations $\leq \mathbf{1}$ \\
\hline $\mathbf{2 a}$ & 81.51 & 79.67 & 7 & 7 & 0 & 4.38 & 444.15 & 0 \\
$\mathbf{2 b}$ & 78.32 & 88.90 & 8 & 8 & 0 & 4.43 & 472.16 & 0 \\
$\mathbf{3 a}$ & 71.57 & 108.48 & 6 & 8 & 3 & 2.93 & 442.16 & 0 \\
$\mathbf{3 b}$ & 68.38 & 117.72 & 7 & 9 & 3 & 2.98 & 472.49 & 0 \\
$\mathbf{4}$ & 79.08 & 83.80 & 3 & 7 & 2 & 2.34 & 336.35 & 0 \\
$\mathbf{5 a}$ & 79.02 & 86.87 & 5 & 8 & 1 & 2.64 & 412.82 & 0 \\
$\mathbf{5 b}$ & 79.02 & 86.87 & 6 & 8 & 1 & 2.91 & 426.10 & 0 \\
$\mathbf{6 a}$ & 77.91 & 90.11 & 6 & 9 & 1 & 2.97 & 461.51 & 0 \\
$\mathbf{6 b}$ & 76.79 & 93.35 & 6 & 10 & 1 & 1.96 & 476.52 & 0 \\
$\mathbf{6 c}$ & 77.91 & 90.11 & 7 & 9 & 1 & 3.24 & 475.53 & 0 \\
$\mathbf{6 d}$ & 76.79 & 93.35 & 7 & 10 & 1 & 2.23 & 490.55 & 0 \\
$\mathbf{7 a}$ & 77.91 & 90.11 & 6 & 9 & 1 & 2.97 & 461.51 & 0 \\
$\mathbf{7 b}$ & 76.79 & 93.35 & 6 & 10 & 1 & 1.96 & 476.52 & 0 \\
$\mathbf{7 c}$ & 77.91 & 90.11 & 7 & 9 & 1 & 3.24 & 475.53 & 0 \\
$\mathbf{7 d}$ & 76.79 & 93.35 & 7 & 10 & 1 & 2.23 & 490.55 & 0 \\
$\mathbf{8}$ & 80.65 & 82.17 & 3 & 8 & 1 & 2.61 & 375.38 & 0 \\
$\mathbf{9}$ & 71.13 & 109.74 & 4 & 10 & 0 & 1.67 & 417.37 & 0 \\
\hline
\end{tabular}

The most relevant descriptors derived for modeling the antitumor activity are listed in Table 3 and the relation between the obtained results of experimentally observed and predicted values of antitumor activity were presented in Table 4. 2D-QSAR models were validated with the leave one out (LOO) method. The best derived QSAR model for the newly synthesized derivatives was presented by the following triparametric equation with correlation coefficient $\left(\mathrm{R}^{2}\right)=0.92$ :

$$
\begin{aligned}
{\left[\mathrm{IC}_{50}=-617.62254+757.93323\right.} & \times \\
& \times \text { PEOE_VSA_FHYD }+306.47775 \times \mathrm{glob}-4.31115
\end{aligned}
$$

where $n=9$, RMSE $=3.4641, R^{2}=0.92722, n$ : number of compounds used for construction of model; RMSE: Root mean square error; $R^{2}$ : correlation coefficient.

From this model, it was observed that PEOE_VSA_FHYD (Fractional hydrophobic Van der Waal surface area) and glob (Globularity, or inverse condition number (smallest eigenvalue divided by the largest eigen value) of the covariance matrix of atomic coordinates. A value of 1 indicates a perfect sphere while a value of 0 indicates a two- or one-dimensional object) were positively correlated with antitumor activity but MNDO_dipole (The dipole moment calculated using the MNDO Hamiltonian [MOPAC]) was negatively correlated with activity indicated that increasing in the values PEOE_VSA_FHYD is beneficial for antitumor activity and the experimental $\mathrm{IC}_{50}$ was matched with the expected $\mathrm{IC}_{50}$. 
Table 3. The most relevant discriptors derived for modeling the antitumor activity.

\begin{tabular}{ccccc}
\hline Compd. No & $\boldsymbol{P}_{\text {IC }} \mathbf{5 0}$ & PEOE_VS & Glob & MNDO_di \\
\hline $\mathbf{4}$ & 36.2 & 0.8547 & 0.0796 & 3.7779 \\
$\mathbf{5 a}$ & 10.6 & 0.8258 & 0.0670 & 4.2328 \\
$\mathbf{5 b}$ & 32.8 & 0.8332 & 0.0725 & 4.3450 \\
$\mathbf{6 a}$ & 26.4 & 0.8517 & 0.0514 & 1.6865 \\
$\mathbf{6 b}$ & 36.0 & 0.8602 & 0.0519 & 0.0000 \\
$\mathbf{6 c}$ & 39.9 & 0.8571 & 0.1028 & 5.6151 \\
$\mathbf{6 d}$ & 43.6 & 0.8650 & 0.0387 & 1.9895 \\
$\mathbf{7 a}$ & 21.6 & 0.8517 & 0.0389 & 5.1133 \\
$\mathbf{7 b}$ & 26.8 & 0.8602 & 0.0591 & 5.0499 \\
$\mathbf{7 c}$ & 36.5 & 0.8517 & 0.0690 & 3.9824 \\
\hline
\end{tabular}

Table 4. Experimentally observed, predicted activity of the new compounds and their residual.

\begin{tabular}{cccc}
\hline Compd. No & Activity $\boldsymbol{P}$ IC $_{\mathbf{5 0}}$ & \$PRED & \$RES \\
\hline $\mathbf{4}$ & 36.2 & 38.2548 & -2.0548 \\
$\mathbf{5 a}$ & 10.6 & 10.5372 & 0.0628 \\
$\mathbf{5 b}$ & 32.8 & & \\
$\mathbf{6 a}$ & 26.4 & 36.3854 & -9.9854 \\
$\mathbf{6 b}$ & 36.0 & 50.2617 & -14.2617 \\
$\mathbf{6 c}$ & 39.9 & 39.2977 & 0.6023 \\
$\mathbf{6 d}$ & 43.6 & 41.2839 & 2.3161 \\
$\mathbf{7 a}$ & 21.6 & 17.7661 & 3.8339 \\
$\mathbf{7 b}$ & 26.8 & 30.6762 & -3.8762 \\
$\mathbf{7 c}$ & 36.5 & 35.9551 & 0.5448 \\
\hline
\end{tabular}

\section{Experimental}

\subsection{General Information}

Unless specified all chemicals were of commercial grade, obtained from Aldrich Chemical Co. (Milwaukee, WI, USA), and used without further purification. Melting points were carried out by the open capillary tube method using a Gallenkamp digital melting point Griffin apparatus 1901 and they were uncorrected. Elemental microanalyses were recorded at the Regional Center of Mycology and Biotechnology, Al-Azhar University. Infrared Spectra were recorded on Shimadzu infrared spectrophotometer IR Affinity-1 (FTIR-8400S, Kyoto, Japan) and expressed in wave number $\left(\mathrm{cm}^{-1}\right)$, using potassium bromide discs. NMR spectra were recorded on a Varian Mercury VX 300 spectrometer $\left({ }^{1} \mathrm{H}: 300,{ }^{13} \mathrm{C}\right.$ APT: $\left.75 \mathrm{MHz}\right)$ or a $400 \mathrm{MHz}$ high performance digital FT-NMR spectrophotometer using TMS as an internal standard. The exchangeable protons $\left(\mathrm{NH}\right.$, and $\mathrm{OH}$ ) were exchanged by $\mathrm{D}_{2} \mathrm{O}$. Mass Spectra was recorded on Shimadzu QP-2010 plus. All reactions were monitored by thin layer chromatography. Silica gel/TLC-cards DC-Alufolien-Kieselgel with fluorescent indicator $254 \mathrm{~nm}$; layer thickness $0.2 \mathrm{~mm} ; 20 \times 20 \mathrm{~cm}$ aluminum cards were used. Petroleum ether/ethyl acetate $(1: 1)$ or (1:2) was the adopted solvent system. Compounds 1a,b [26,27] were prepared according to reported procedures. 


\subsection{Chemistry}

\subsubsection{General Procedure for the Preparation of $\mathbf{2 a}, \mathbf{b}$}

A mixture of 1a,b (10 mmol) and methyl 2-amino-4,5-dimethoxybenzoate (12 mmol, $2.52 \mathrm{~g})$, freshly prepared fused sodium acetate $(0.3 \mathrm{~g})$ was heated in a boiling water bath in glacial acetic acid $(10 \mathrm{~mL})$ for $3 \mathrm{~h}$. The crystalline product separated on cooling was filtered, washed with water, dried, and recrystallized from ethanol.

Methyl 2-(4-benzylidene-5-oxo-2-phenyl-4,5-dihydroimidazol-1-yl)-4,5-dimethoxybenzoate (2a). Yield: 81\%; mp 221-223 ${ }^{\circ} \mathrm{C}$; IR (KBr, cm ${ }^{-1}$ ): 3086 (CH aromatic), 2958, 2935 (CH aliphatic), 1693, 1674 $\left.(\mathrm{C}=\mathrm{O}) ;{ }^{1} \mathrm{H}-\mathrm{NMR}\left(\mathrm{DMSO}_{-}\right)_{6}\right): \delta 3.65\left(\mathrm{~s}, 3 \mathrm{H}, \mathrm{COOCH}_{3}\right), 3.77\left(\mathrm{~s}, 3 \mathrm{H}, \mathrm{OCH}_{3}\right), 3.88\left(\mathrm{~s}, 3 \mathrm{H}, \mathrm{OCH}_{3}\right)$, 7.36-7.43 (m, 4H, benzyl. 3,4,5-CHs + benzo.6-CH) 7.54-7.63 (m, 6H, phenyl CHs + benzo.3-CH), 8.04 (d, 2H, $J=6.9$, benzyl. 2,6-CHs), 8.57 (s, 1H, CH); ${ }^{13} \mathrm{C}-\mathrm{NMR}$ (DMSO): 52.4, 56.09, 56.1, 103.4, 107.1, 112.6, 128.4, 128.7, 129.1, 129.7, 130.0, 130.4, 131.9, 132.3, 134.0, 137.1, 144.2, 154.0, 163.9, 167.4, 167.8; MS, $m / z(\%): \mathrm{M}^{+}, 442(23.54 \%), 105(100 \%)$. Anal. Calcd. for $\mathrm{C}_{26} \mathrm{H}_{22} \mathrm{~N}_{2} \mathrm{O}_{5}(442.46)$ : C, 70.58; H, 5.01; N, 6.33. Found: C, 70.32; H, 5.12; N, 6.44\%.

Methyl 4,5-dimethoxy-2-(4-(4-methoxybenzylidene)-5-oxo-2-phenyl-4,5-dihydro-1H-imidazol-1-yl)benzoate (2b). Yield: 84\%; mp 211-212 ${ }^{\circ} \mathrm{C}$; IR (KBr, $\left.\mathrm{cm}^{-1}\right)$ : 3062 (CH aromatic), 2939, $2839(\mathrm{CH}$ aliphatic), 1720, $1678(\mathrm{C}=\mathrm{O})$; ${ }^{1} \mathrm{H}-\mathrm{NMR}\left(\mathrm{DMSO}-d_{6}\right): \delta 3.63\left(\mathrm{~s}, 3 \mathrm{H}, \mathrm{COOCH}_{3}\right), 3.84\left(\mathrm{~s}, 6 \mathrm{H}, 2 \mathrm{OCH}_{3}\right)$, $3.86\left(\mathrm{~s}, 3 \mathrm{H}, \mathrm{OCH}_{3}\right), 6.95(\mathrm{~d}, 2 \mathrm{H}, J=9$, benzyl. 3, 5-CHs), $7.39(\mathrm{~s}, 1 \mathrm{H}$, benzo.6-CH) 7.54-7.63 (m, 6H, phenyl CHs + benzo.3-CH), $8.04\left(\mathrm{~d}, 2 \mathrm{H}, J=6.9\right.$, benzyl. 2,6-CHs), $8.58(\mathrm{~s}, 1 \mathrm{H}, \mathrm{CH}) ;{ }^{13} \mathrm{C}-\mathrm{NMR}$ (DMSO): 51.8, 55.5, 55.7, 56.1, 102.7, 106.4, 112.5, 114.4, 126.0, 127.6, 128.2, 130.5, 131.4, 131.7, $133.5,134.2,136.7,143.5,153.4,160.0,163.5,166.8,167.2 ; \mathrm{MS}, m / z(\%): \mathrm{M}^{+}, 472(68.44 \%), 105$ (100\%). Anal. Calcd. for $\mathrm{C}_{27} \mathrm{H}_{24} \mathrm{~N}_{2} \mathrm{O}_{6}$ (472.48): C, 68.63; H, 5.12; N, 5.93. Found: C, 68.85; H, 5.00; $\mathrm{N}, 6.01 \%$.

\subsubsection{General Procedure for the Preparation of $\mathbf{3 a}, \mathbf{b}$}

A solution of $\mathbf{2 a}, \mathbf{b}(10 \mathrm{mmol})$, hydrazine hydrate $(12 \mathrm{mmol}, 0.60 \mathrm{~g})$ in methanol $(50 \mathrm{~mL})$ was refluxed for $2 \mathrm{~h}$. The mixture was concentrated under vacuum, and water was added to the residue, which was filtered, dried, and crystallized from ethanol.

2-(4-Benzylidene-5-oxo-2-phenyl-4,5-dihydroimidazol-1-yl)-4,5-dimethoxybenzo- hydrazide (3a). Yield: 71\%; mp 151-153 ${ }^{\circ} \mathrm{C}$; IR (KBr, $\left.\mathrm{cm}^{-1}\right)$ : 3456, 3356, $3305\left(\mathrm{NH}_{2}, \mathrm{NH}\right), 3059$ (CH aromatic), 2997, 2951 (CH aliphatic), $1674(\mathrm{C}=\mathrm{Os})$; ${ }^{1} \mathrm{H}-\mathrm{NMR}\left(\mathrm{DMSO}-d_{6}\right): \delta 3.73\left(\mathrm{~s}, 3 \mathrm{H}, \mathrm{OCH}_{3}\right), 3.79(\mathrm{~s}, 3 \mathrm{H}$, $\left.\mathrm{OCH}_{3}\right), 6.45\left(\mathrm{~s}, 2 \mathrm{H}, \mathrm{NH}_{2}\right), 7.11-7.38(\mathrm{~m}, 4 \mathrm{H}$, benzyl. 3,4,5-CHs + benzo.6-CH), 7.42-7.49 (m, 6H, phenyl CHs + benzo.3-CH), $7.79(\mathrm{~d}, 2 \mathrm{H}, J=6.9$, benzyl. 2,6-CHs), $8.59(\mathrm{~s}, 1 \mathrm{H}, \mathrm{CH}), 9.33(\mathrm{~s}, 1 \mathrm{H}$, $\mathrm{NH}) ;{ }^{13} \mathrm{C}-\mathrm{NMR}$ (DMSO): 55.1, 55.9, 99.0, 112.2, 126.1, 127.3, 127.9, 128.0, 129.0, 131.1, 133.9, 138.2, 139.2, 143.5, 148.2, 154.7, 166.0, 167.2, 170.5; MS, m/z (\%): $\mathrm{M}^{+}, 442$ (0.25\%), 50 (100\%). Anal. Calcd. for $\mathrm{C}_{25} \mathrm{H}_{22} \mathrm{~N}_{4} \mathrm{O}_{4}$ (442.46): C, 67.86; H, 5.01; N, 12.66. Found: C, 67.86; H, 5.12; N, 12.93\%. 
2-(4-(4-Methoxybenzylidene)-5-oxo-2-phenyl-4,5-dihydroimidazol-1-yl)-4,5-dimethoxybenzohydrazide (3b). Yield: 74\%; mp 201-203 ${ }^{\circ} \mathrm{C}$; IR (KBr, cm $\left.{ }^{-1}\right)$ : 3441, 3244, $3200\left(\mathrm{NH}_{2}, \mathrm{NH}\right), 3050$ (CH aromatic), 2924, 2835 (CH aliphatic), 1674, 1658 (C=Os); ${ }^{1} \mathrm{H}-\mathrm{NMR}\left(\mathrm{DMSO}-d_{6}\right): \delta 3.71\left(\mathrm{~s}, 3 \mathrm{H}, \mathrm{OCH}_{3}\right), 3.80$ (s, $\left.3 \mathrm{H}, \mathrm{OCH}_{3}\right), 3.86\left(\mathrm{~s}, 3 \mathrm{H}, \mathrm{OCH}_{3}\right), 6.88\left(\mathrm{~s}, 2 \mathrm{H}, \mathrm{NH}_{2}\right), 6.98$ (d, 2H, J = 8.7, benzyl. 3, 5-CHs), 7.39 (s, $1 \mathrm{H}$, benzo.6-CH), 7.55-7.63 (m, 6H, phenyl CHs + benzo.3-CH), $8.04(\mathrm{~d}, 2 \mathrm{H}, J=6.9$, benzyl. 2,6-CHs), 8.60 (s, 1H, CH), 10.30 (s, 1H, NH); ${ }^{13} \mathrm{C}-\mathrm{NMR}$ (DMSO): 51.8, 55.1, 55.3, 102.5, 112.8, $125.7,126.4,127.7,128.3,130.0,131.4,132.6,133.1,136.0,143.4,153.1,154.7,160.05,163.5$, 167.2, 167.5; MS, $m / z(\%): \mathrm{M}^{+}, 472(0.38 \%), 57$ (100\%). Anal. Calcd. for $\mathrm{C}_{26} \mathrm{H}_{24} \mathrm{~N}_{4} \mathrm{O}_{5}$ (472.49): C, 66.09; H, 5.12; N, 11.86. Found: C, 66.34; H, 5.45; N, 12.24\%.

\subsubsection{Synthesis of 4-Amino-7,8-dimethoxy-1-phenylimidazo[1,5-a]quinazolin-5(4H)-one (4)}

To a suspension of the appropriate $\mathbf{2 a , b}(10 \mathrm{mmol})$ in aqueous ethanol $(80 \%)$, hydrazine hydrate ( $30 \mathrm{mmol}, 1.50 \mathrm{~g}$ ) was added. The mixture was refluxed for $24 \mathrm{~h}$. The resulting solution was distilled under vacuum and the residue was triturated with ice water. The separated solid was filtered, washed with water, and crystallized from ethanol. Yield: $64 \%$ from $\mathbf{2 a}, 72 \%$ from $\mathbf{2 b}$; mp $281-283{ }^{\circ} \mathrm{C}$; IR $\left(\mathrm{KBr}, \mathrm{cm}^{-1}\right)$ : 3317, $3267\left(\mathrm{NH}_{2}\right), 3074(\mathrm{CH}$ aromatic), 2927, 2839 ( $\mathrm{CH}$ aliphatic), $1680(\mathrm{C}=\mathrm{O})$; ${ }^{1} \mathrm{H}-\mathrm{NMR}\left(\mathrm{DMSO}-d_{6}\right): \delta 3.89\left(\mathrm{~s}, 3 \mathrm{H}, \mathrm{OCH}_{3}\right), 3.90\left(\mathrm{~s}, 3 \mathrm{H}, \mathrm{OCH}_{3}\right), 4.42\left(\mathrm{~s}, 2 \mathrm{H}, \mathrm{NH}_{2}\right), 7.19(\mathrm{~s}, 1 \mathrm{H}$, imidazo.9-CH), 7.45-7.55 (m, 7H, Ar-H); ${ }^{13} \mathrm{C}-\mathrm{NMR}$ (DMSO): 55.7, 55.9, 105.0, 107.9, 113.1, 127.8, 128.0, 128.3, 129.5, 135.1, 143.9, 148.7, 154.5, 154.7, 160.3, 166.3; MS, $m / z(\%): \mathrm{M}^{+}+1,337(0.25 \%)$, 196 (100\%). Anal. Calcd. for $\mathrm{C}_{18} \mathrm{H}_{16} \mathrm{~N}_{4} \mathrm{O}_{3}$ (336.34): C, 64.28; H, 4.79; N, 16.66. Found: C, 64.37; H, $4.86 ; \mathrm{N}, 16.92 \%$.

\subsubsection{General Procedure for the Preparation of 5a,b}

A solution of compound 4 ( $5 \mathrm{mmol}, 1.68 \mathrm{~g})$ in dry DMF $(5 \mathrm{~mL})$ containing chloroacetylchloride or chloropropionyl chloride $(5.5 \mathrm{mmol})$ was stirred at room temperature $\left(25-30^{\circ} \mathrm{C}\right)$ for $24 \mathrm{~h}$. The solution was poured onto crushed ice and the resulting solid was filtered, washed and crystallized from ethanol.

2-Chloro-N-(7,8-dimethoxy-5-oxo-1-phenylimidazo[1,5-a]quinazolin-5(4H)-yl) acetamide (5a). Yield: 82\%; mp 176-178 ${ }^{\circ} \mathrm{C}$; IR (KBr, cm ${ }^{-1}$ ): $3224(\mathrm{NH}), 3035$ (CH aromatic), 2947, 2854 (CH aliphatic), $1674(\mathrm{C}=\mathrm{O}) ;{ }^{1} \mathrm{H}-\mathrm{NMR}\left(\mathrm{DMSO}-d_{6}\right): \delta 3.81\left(\mathrm{~s}, 3 \mathrm{H}, \mathrm{OCH}_{3}\right), 3.86\left(\mathrm{~s}, 3 \mathrm{H}, \mathrm{OCH}_{3}\right), 4.44\left(\mathrm{~s}, 2 \mathrm{H}, \mathrm{CH}_{2}\right)$, 7.40-8.20 (m, 8H, Ar-H), 11.47 (s, 1H, NH) ${ }^{13} \mathrm{C}-\mathrm{NMR}$ (DMSO): 43.3, 55.4, 55.7, 103.3, 107.6, 112.0, 127.3, 128.0, 128.2, 129.0, 135.0, 144.1, 144.2, 153.1, 153.4, 164.9, 167.0; MS, $m / z(\%): \mathrm{M}^{+}+2,414$ (0.31\%), 412 (0.91\%), 105 (100\%). Anal. Calcd. for $\mathrm{C}_{20} \mathrm{H}_{17} \mathrm{ClN}_{4} \mathrm{O}_{4}(412.82): \mathrm{C}, 58.19 ; \mathrm{H}, 4.15 ; \mathrm{N}$, 13.57. Found: C, 58.34; H, 4.01; N, 13.78\%.

3-Chloro-N-(7,8-dimethoxy-5-oxo-1-phenylimidazo[1,5-a]quinazolin-5(4H)-yl) propamide $\quad(\mathbf{5 b})$. Yield: 75\%; mp 209-210 ${ }^{\circ} \mathrm{C}$; IR $\left(\mathrm{KBr}, \mathrm{cm}^{-1}\right)$ : $3248(\mathrm{NH}), 3055$ (CH aromatic), 2951, $2824(\mathrm{CH}$ aliphatic), $1670(\mathrm{C}=\mathrm{O}) ;{ }^{1} \mathrm{H}-\mathrm{NMR}$ (DMSO- $\left.d_{6}\right): \delta 2.88\left(\mathrm{t}, 2 \mathrm{H}, \mathrm{CH}_{2}, J=7.8\right), 3.79\left(\mathrm{t}, 2 \mathrm{H}, \mathrm{CH}_{2}, J=7.8\right.$ ), $3.90\left(\mathrm{~s}, 3 \mathrm{H}, \mathrm{OCH}_{3}\right), 3.92\left(\mathrm{~s}, 3 \mathrm{H}, \mathrm{OCH}_{3}\right), 7.15-8.60(\mathrm{~m}, 8 \mathrm{H}, \mathrm{Ar}-\mathrm{H}), 10.7(\mathrm{~s}, 1 \mathrm{H}, \mathrm{NH}) ;{ }^{13} \mathrm{C}-\mathrm{NMR}$ (DMSO): 36.0, 52.2, 54.4, 55.7, 103.9, 108.2, 112.9, 127.1, 128.0, 128.2, 128.9,131.5, 133.3, 135.0, 
137.5, 143.8, 152.8, 166.7, 170.4; MS, m/z (\%): $\mathrm{M}^{+}, 426(0.22 \%), 104$ (100\%). Anal. Calcd. for $\mathrm{C}_{21} \mathrm{H}_{19} \mathrm{ClN}_{4} \mathrm{O}_{4}$ (426.85): C, 59.09; H, 4.49; N, 13.13. Found: C, 59.18; H, 4.53; N, 13.47\%.

\subsubsection{General Procedure for the Preparation of $\mathbf{6 a}-\mathbf{d}$}

A mixture of equimolar amounts of the appropriate $\mathbf{5 a}, \mathbf{b}$ and the corresponding secondary amine $(2 \mathrm{mmol})$ in dry acetonitrile $(30 \mathrm{~mL})$ containing potassium carbonate $(4 \mathrm{mmol}, 0.55 \mathrm{~g})$ was refluxed for $12 \mathrm{~h}$ and the reaction mixture was filtered hot. The solid which was separated upon storing the clear reaction mixture at room temperature overnight was collected and crystallized from ethanol.

N-(7,8-Dimethoxy-5-oxo-1-phenylimidazo[1,5-a]quinazolin-5(4H)-yl)-2-(piperidin-1-yl)acetamide (6a). Yield: 77\%; mp 186-189 ${ }^{\circ} \mathrm{C}$; IR ( $\left.\mathrm{KBr}, \mathrm{cm}^{-1}\right)$ : $3217(\mathrm{NH}), 3016(\mathrm{CH}$ aromatic), 2943, $2879(\mathrm{CH}$ aliphatic), 1693, $1670(\mathrm{C}=\mathrm{Os}) ;{ }^{1} \mathrm{H}-\mathrm{NMR}$ (DMSO- $\left.d_{6}\right): \delta 1.42-1.43\left(\mathrm{~m}, 2 \mathrm{H}, \mathrm{CH}_{2}\right), 1.63-1.68(\mathrm{~m}, 4 \mathrm{H}$, $\left.2 \mathrm{CH}_{2}\right), 2.46\left(\mathrm{t}, 4 \mathrm{H}, 2 \mathrm{CH}_{2}, J=8.7\right), 3.07\left(\mathrm{~s}, 2 \mathrm{H}, \mathrm{CH}_{2}\right), 3.80\left(\mathrm{~s}, 3 \mathrm{H}, \mathrm{OCH}_{3}\right), 3.86\left(\mathrm{~s}, 3 \mathrm{H}, \mathrm{OCH}_{3}\right)$, 7.40-8.40 (m, 8H, Ar-H), $11.8(\mathrm{~s}, 1 \mathrm{H}, \mathrm{NH}) ;{ }^{13} \mathrm{C}-\mathrm{NMR}$ (DMSO): 23.4, 25.2, 52.2, 55.4, 55.4, 62.9, $102.9,106.8,112.1,127.4,128.1,128.3,129.0,135.9,144.3,144.4,153.1,153.4,166.5,169.6$; MS, $m / z(\%): \mathrm{M}^{+}, 461$ (47.5\%), 254 (100\%). Anal. Calcd. for $\mathrm{C}_{25} \mathrm{H}_{27} \mathrm{~N}_{5} \mathrm{O}_{4}$ (461.51): C, 65.06; H, 5.90; N, 15.17. Found: C, 65.38; H, 5.93; N, 15.42\%.

N-(7,8-Dimethoxy-5-oxo-1-phenylimidazo[1,5-a]quinazolin-5(4H)-yl)-2-(4-methylpiperazin-1-yl)acetamide (6b).Yield: 81\%; mp 167-168 ${ }^{\circ} \mathrm{C}$; IR (KBr, cm ${ }^{-1}$ ): 3205 (NH), 3030 (CH aromatic), 2985, 2947, 2816 (CH aliphatic), 1689, $1670(\mathrm{C}=\mathrm{Os}) ;{ }^{1} \mathrm{H}-\mathrm{NMR}\left(\mathrm{DMSO}-d_{6}\right): \delta 2.19\left(\mathrm{~s}, 3 \mathrm{H}, \mathrm{CH}_{3}\right), 2.46-2.53(\mathrm{~m}, 8 \mathrm{H}$, $\left.4 \mathrm{CH}_{2}\right), 3.75\left(\mathrm{~s}, 2 \mathrm{H}, \mathrm{CH}_{2}\right), 3.80\left(\mathrm{~s}, 3 \mathrm{H}, \mathrm{OCH}_{3}\right), 3.86\left(\mathrm{~s}, 3 \mathrm{H}, \mathrm{OCH}_{3}\right), 7.18-8.41(\mathrm{~m}, 8 \mathrm{H}, \mathrm{Ar}-\mathrm{H}), 11.76(\mathrm{~s}$, $1 \mathrm{H}, \mathrm{NH}) ;{ }^{13} \mathrm{C}-\mathrm{NMR}$ (DMSO): 46.2, 52.5, 53.3, 54.7, 54.9, 56.0, 56.1, 62.5, 103.6, 107.5, 112.7, 127.9, 128.6, 129.05, 136.4, 144.0, 153.7, 167.0, 169.8; MS, m/z (\%): $\mathrm{M}^{+}, 476$ (19.9\%), 55 (100\%). Anal. Calcd. for $\mathrm{C}_{25} \mathrm{H}_{28} \mathrm{~N}_{6} \mathrm{O}_{4}$ (476.52): C, 63.01; H, 5.92; N, 17.64. Found: C, 63.13; H, 6.12; N, 17.79\%.

$\mathrm{N}$-(7,8-Dimethoxy-5-oxo-1-phenylimidazo[1,5-a]quinazolin-5(4H)-yl)-3-(piperi-din-1-yl)propamide (6c). Yield: 66\%; mp 105-108 ${ }^{\circ} \mathrm{C}$; IR $\left(\mathrm{KBr}, \mathrm{cm}^{-1}\right)$ : $3263(\mathrm{NH}), 3010(\mathrm{CH}$ aromatic), 2931, $2894(\mathrm{CH}$ aliphatic), 1674 (br., $\mathrm{C}=\mathrm{O})$ ) ${ }^{1} \mathrm{H}-\mathrm{NMR}\left(\mathrm{DMSO}-d_{6}\right): \delta 1.37-1.48\left(\mathrm{~m}, 6 \mathrm{H}, 3 \mathrm{CH}_{2}\right), 2.53-2.55(\mathrm{~m}, 4 \mathrm{H}$, $\left.2 \mathrm{CH}_{2}\right), 2.61\left(\mathrm{t}, 2 \mathrm{H}, 2 \mathrm{CH}_{2}, J=6.3\right), 3.69$ (t, $\left.2 \mathrm{H}, 2 \mathrm{CH}_{2}, J=9.3\right), 3.90\left(\mathrm{~s}, 3 \mathrm{H}, \mathrm{OCH}_{3}\right), 3.91\left(\mathrm{~s}, 3 \mathrm{H}, \mathrm{OCH}_{3}\right)$, 7.20-8.10 (m, 8H, Ar-H), 10.85 (s, 1H, NH); ${ }^{13} \mathrm{C}-\mathrm{NMR}$ (DMSO): 22.2, 25.6, 35.2, 52.6, 53.7, 54.4, 55.9, 56.0, 56.1, 104.6, 108.4, 112.6, 127.4, 127.9, 128.2, 128.6, 128.7, 129.1, 136.3, 144.2, 153.6, 167.6, 170.7; MS, $m / z(\%): \mathrm{M}^{+}, 475$ (0.5\%), 98 (100\%). Anal. Calcd. for $\mathrm{C}_{26} \mathrm{H}_{29} \mathrm{~N}_{5} \mathrm{O}_{4}$ (475.53): C, $65.67 ; \mathrm{H}, 6.15 ; \mathrm{N}, 14.73$. Found: C, 65.46; H, 5.84; N, 14.50\%.

N-(7,8-Dimethoxy-5-oxo-1-phenylimidazo[1,5-a]quinazolin-5(4H)-yl)-3-(4-methylpiperazin-1-yl)propamide (6d). Yield: 69\%; mp 130-132 ${ }^{\circ} \mathrm{C}$; IR $\left(\mathrm{KBr}, \mathrm{cm}^{-1}\right)$ : 3217 (NH), 3059 (CH aromatic), 2939, 2835 (CH aliphatic), 1674 (br., $\mathrm{C}=\mathrm{O}) ;{ }^{1} \mathrm{H}-\mathrm{NMR}\left(\mathrm{DMSO}-d_{6}\right): \delta 2.12\left(\mathrm{~s}, 3 \mathrm{H}, \mathrm{CH}_{3}\right), 2.28-2.45(\mathrm{~m}, 8 \mathrm{H}$, $\left.4 \mathrm{CH}_{2}\right), 2.62\left(\mathrm{t}, 2 \mathrm{H}, 2 \mathrm{CH}_{2}, J=6.3\right), 3.72\left(\mathrm{t}, 2 \mathrm{H}, \mathrm{CH}_{2}, J=7.8\right), 3.90\left(\mathrm{~s}, 3 \mathrm{H}, \mathrm{OCH}_{3}\right), 3.92\left(\mathrm{~s}, 3 \mathrm{H}, \mathrm{OCH}_{3}\right)$, 7.19-8.24 (m, 8H, Ar-H), 10.84 (s, 1H, NH); ${ }^{13} \mathrm{C}-\mathrm{NMR}$ (DMSO): 34.4, 44.5, 51.1, 52.8, 54.3, 55.3, $103.9,106.2$, 111.7, 127.1, 128.3, 128.8, 129.5, 135.1, 143.5, 144.4, 152.7, 153.4, 167.0, 170.4; MS, 
$m / z(\%): \mathrm{M}^{+}, 490(0.11 \%), 70(100 \%)$. Anal. Calcd. for $\mathrm{C}_{26} \mathrm{H}_{30} \mathrm{~N}_{6} \mathrm{O}_{4}$ (490.55): C, 63.66; H, 6.16; N, 17.13. Found: $\mathrm{C}, 64.13 ; \mathrm{H}, 6.28 ; \mathrm{N}, 17.46 \%$.

\subsubsection{General Procedure for the Preparation of $7 \mathbf{a}-\mathbf{d}$}

A mixture of equimolar amounts of compound 4 and the corresponding 2/3-chloro-1-(piperidin-1yl/4-methylpiperazin-1-yl) ethanone/propan-1-one $(2 \mathrm{mmol})$ in dry DMF $(10 \mathrm{~mL})$ containing triethylamine $(0.5 \mathrm{~mL})$ was refluxed for $10 \mathrm{~h}$. The resulting solution was cooled and poured onto crushed ice. The separated solid was filtered, dried, and crystallized from methanol.

7,8-Dimethoxy-4-(2-oxo-2-piperidin-1-yl)ethylamino)-1-phenylimidazo[1,5-a] quinazolin-5(4H)-one (7a). Yield: 77\%; mp 142-143 ${ }^{\circ} \mathrm{C}$; IR ( $\left.\mathrm{KBr}, \mathrm{cm}^{-1}\right)$ : $3232(\mathrm{NH}), 3056(\mathrm{CH}$ aromatic), 2923, $2854(\mathrm{CH}$ aliphatic), 1670, $1654(\mathrm{C}=\mathrm{O}) ;{ }^{1} \mathrm{H}-\mathrm{NMR}$ (DMSO- $\left.d_{6}\right): \delta 1.27-1.38\left(\mathrm{~m}, 2 \mathrm{H}, \mathrm{CH}_{2}\right), 1.42-1.56(\mathrm{~m}, 4 \mathrm{H}$, $\left.2 \mathrm{CH}_{2}\right), 3.11-3.28\left(\mathrm{~m}, 4 \mathrm{H}, 2 \mathrm{CH}_{2}\right), 3.73\left(\mathrm{~s}, 2 \mathrm{H}, \mathrm{CH}_{2}\right), 3.89\left(\mathrm{~s}, 3 \mathrm{H}, \mathrm{OCH}_{3}\right), 3.91\left(\mathrm{~s}, 3 \mathrm{H}, \mathrm{OCH}_{3}\right), 6.45(\mathrm{~s}$, $1 \mathrm{H}, \mathrm{NH}), 7.17-8.87$ (m, 8H, Ar-H); ${ }^{13} \mathrm{C}-\mathrm{NMR}$ (DMSO): 21.3, 21.8, 45.2, 51.0, 55.1, 55.7, 98.9, 99.6, $112.0,127.2$, 128.1, 128.2, 128.8, 131.5, 139.2, 147.7, 153.4, 154.6, 167.4; MS, $m / z(\%): \mathrm{M}^{+}, 461$ (0.51\%), 196 (100\%). Anal. Calcd. for $\mathrm{C}_{25} \mathrm{H}_{27} \mathrm{~N}_{5} \mathrm{O}_{4}$ (461.51): C, 65.06; H, 5.90; N, 15.17. Found: C, $65.11 ; \mathrm{H}, 5.97 ; \mathrm{N}, 15.24 \%$.

7,8-Dimethoxy-4-(2-(4-methylpiperazin-1-yl)-2-oxo-ethylamino)-1-phenylimidazo[1,5-a]quinazolin5(4H)-one (7b). Yield: 67\%; mp 160-161 ${ }^{\circ} \mathrm{C}$; IR $\left(\mathrm{KBr}, \mathrm{cm}^{-1}\right): 3305(\mathrm{NH}), 3059$ (CH aromatic), 2924, $2854\left(\mathrm{CH}\right.$ aliphatic), 1670, $1661(\mathrm{C}=\mathrm{O}) ;{ }^{1} \mathrm{H}-\mathrm{NMR}$ (DMSO- $\left.d_{6}\right): \delta 2.22-2.34\left(\mathrm{~m}, 7 \mathrm{H}, \mathrm{CH}_{3}\right.$ and $\left.2 \mathrm{CH}_{2}\right)$, $3.16\left(\mathrm{t}, 4 \mathrm{H}, 2 \mathrm{CH}_{2}, J=6.3\right), 3.507\left(\mathrm{~s}, 2 \mathrm{H}, \mathrm{CH}_{2}\right), 3.89\left(\mathrm{~s}, 3 \mathrm{H}, \mathrm{OCH}_{3}\right), 3.90\left(\mathrm{~s}, 3 \mathrm{H}, \mathrm{OCH}_{3}\right), 6.44(\mathrm{~s}, 1 \mathrm{H}$, $\mathrm{NH}), 7.09-8.09$ (m, 8H, Ar-H); ${ }^{13} \mathrm{C}-\mathrm{NMR}$ (DMSO): 44.5, 51.2, 52.1, 53.5, 54.3, 55.3, 103.9, 106.4, $111.8,127.2,128.3,128.8,129.3,130.1,135.2,143.6,144.7,152.7,153.4,167.0,170.4 ; \mathrm{MS}, \mathrm{m} / z(\%)$ : $\mathrm{M}^{+}, 476$ (0.22\%), 105 (100\%). Anal. Calcd. for $\mathrm{C}_{25} \mathrm{H}_{28} \mathrm{~N}_{6} \mathrm{O}_{4}$ (476.52): C, 63.01; H, 5.92; N, 17.64. Found: C, 62.98; H, 6.23; N, 17.81\%.

7,8-Dimethoxy-4-(3-oxo-2-piperidin-1-yl)propylamino)-1-phenylimidazo[1,5-a]quinazolin-5(4H)-one (7c). Yield: 69\%; mp 98-100 ${ }^{\circ} \mathrm{C}$; IR (KBr, cm $\left.{ }^{-1}\right): 3255(\mathrm{NH}), 3028(\mathrm{CH}$ aromatic), 2927, $2854(\mathrm{CH}$ aliphatic), 1672, $1654(\mathrm{C}=\mathrm{O}) ;{ }^{1} \mathrm{H}-\mathrm{NMR}\left(\mathrm{DMSO}-d_{6}\right): \delta 1.19-1.27\left(\mathrm{~m}, 2 \mathrm{H}, \mathrm{CH}_{2}\right), 1.44-1.56(\mathrm{~m}, 4 \mathrm{H}$, $\left.2 \mathrm{CH}_{2}\right), 2.72\left(\mathrm{t}, 2 \mathrm{H}, \mathrm{CH}_{2}, J=11.1\right), 2.97\left(\mathrm{t}, 2 \mathrm{H}, 2 \mathrm{CH}_{2}, J=11.4\right), 3.43-3.69$ (t, 4H, 2CH,$\left.J=6.6\right), 3.81$ $\left(\mathrm{s}, 3 \mathrm{H}, \mathrm{OCH}_{3}\right), 3.91\left(\mathrm{~s}, 3 \mathrm{H}, \mathrm{OCH}_{3}\right), 6.46(\mathrm{~s}, 1 \mathrm{H}, \mathrm{NH}), 7.13-8.08(\mathrm{~m}, 8 \mathrm{H}, \mathrm{Ar}-\mathrm{H}) ;{ }^{13} \mathrm{C}-\mathrm{NMR}$ (DMSO): 23.8, 24.6, 25.9, 44.5, 51.0, 55.1, 55.7, 98.9, 99.6, 112.0, 127.1, 128.1, 128.2, 129.0, 133.2, 139.2, 147.7, 153.4, 154.6, 162.8, 167.4; MS, $m / z(\%): \mathrm{M}^{+}, 475(0.52 \%), 207$ (100\%). Anal. Calcd. for $\mathrm{C}_{26} \mathrm{H}_{29} \mathrm{~N}_{5} \mathrm{O}_{4}$ (475.53): C, 65.67; H, 6.15; N, 14.73. Found: C, 65.78; H, 6.21; N, 14.92\%.

7,8-Dimethoxy-4-(3-(4-methylpiperazin-1-yl)-3-oxo-propylamino)-1-phenylimidazo[1,5-a]quinazolin5(4H)-one (7d). Yield: 69\% mp 210-211 ${ }^{\circ} \mathrm{C}$; IR ( $\left.\mathrm{KBr}, \mathrm{cm}^{-1}\right): 3217(\mathrm{NH}), 3056$ (CH aromatic), 2924, 2850 (CH aliphatic), 1674, $1658(\mathrm{C}=\mathrm{O}) ;{ }^{1} \mathrm{H}-\mathrm{NMR}$ (DMSO- $\left.d_{6}\right): \delta 2.12-2.43\left(\mathrm{~m}, 7 \mathrm{H}, \mathrm{CH}_{3}\right.$ and $\left.2 \mathrm{CH}_{2}\right)$, $2.72\left(\mathrm{t}, 2 \mathrm{H}, \mathrm{CH}_{2}, J=11.1\right), 2.88$ (t, $2 \mathrm{H}, \mathrm{CH}_{2}, J=11.4$ ), 3.05 (t, $4 \mathrm{H}, 2 \mathrm{CH}_{2}, J=7.2$ ), 3.89 (s, $3 \mathrm{H}$, $\left.\mathrm{OCH}_{3}\right), 3.90\left(\mathrm{~s}, 3 \mathrm{H}, \mathrm{OCH}_{3}\right), 6.45(\mathrm{~s}, 1 \mathrm{H}, \mathrm{NH}) 7.19-8.14(\mathrm{~m}, 8 \mathrm{H}, \mathrm{Ar}-\mathrm{H}) ;{ }^{13} \mathrm{C}-\mathrm{NMR}$ (DMSO): 31.1, $36.1,45.8,51.4,55.6,56.4,99.5,100.1,112.8,127.8,127.9,128.6,128.9,129.3,139.8,148.7,155.3$, 
162.8, 167.8; MS, m/z (\%): $\mathrm{M}^{+}, 490$ (22.1\%), 70 (100\%). Anal. Calcd. for $\mathrm{C}_{26} \mathrm{H}_{30} \mathrm{~N}_{6} \mathrm{O}_{4}$ (490.55): C, 63.66; H, 6.16; N, 17.13. Found: C, 63.81; H, 6.30; N, 17.36\%.

3.2.7. Synthesis of 11,12-Dimethoxy-8-phenyl-3,4-dihydro- $2 H$-imidazo[1,5-a][1,2,4]triazino[2,3c] quinazolin-2-one (8)

A mixture of 4 (10 mmol, $3.36 \mathrm{~g}$ ) and 2-chloracetamide (12 mmol, $1.12 \mathrm{~g})$ in dry DMF (15 mL) was refluxed for $24 \mathrm{~h}$. The desired product was obtained through pouring the reaction solution onto ice water and the precipitate was collected, washed, dried, and then crystallized from DMF/water Yield: 61\%; mp 190-192 ${ }^{\circ} \mathrm{C}$; IR (KBr, $\mathrm{cm}^{-1}$ ): $3210(\mathrm{NH}), 3011$ (CH aromatic), 2958, 2924 (CH aliphatic), $1666(\mathrm{C}=\mathrm{O}) ;{ }^{1} \mathrm{H}-\mathrm{NMR}\left(\mathrm{DMSO}-d_{6}\right): \delta 3.74\left(\mathrm{~s}, 2 \mathrm{H}, \mathrm{CH}_{2}\right), 3.89\left(\mathrm{~s}, 3 \mathrm{H}, \mathrm{OCH}_{3}\right), 3.93\left(\mathrm{~s}, 3 \mathrm{H}, \mathrm{OCH}_{3}\right), 5.04$ (s, 1H, NH), 7.29-8.31 (m, 8H, Ar-H); ${ }^{13} \mathrm{C}-\mathrm{NMR}$ (DMSO): 51.5, 55.6, 56.4, 101.4, 112.8, 126.1, $127.9,128.1,128.6,129.2,129.5,131.9,134.2,138.4,146.8,155.3,168.0 ; \mathrm{MS}, \mathrm{m} / z(\%): \mathrm{M}^{+}-1,374$ (8.6\%), 50 (100\%). Anal. Calcd. for $\mathrm{C}_{20} \mathrm{H}_{17} \mathrm{~N}_{5} \mathrm{O}_{3}$ (375.38): C, 63.99; H, 4.56; N, 18.66. Found: C, $63.81 ; \mathrm{H}, 4.78 ; \mathrm{N}, 18.24 \%$.

3.2.8. Synthesis of 1-(7,8-Dimethoxy-5-oxo-1-phenylimidazo[1,5-a]quinazolin-4(5H)-yl)-1Himidazole-2,5-dione (9)

A suspension of $5 \mathbf{a}(5 \mathrm{mmol}, 2.06 \mathrm{~g})$ and potassium cyanate $(10 \mathrm{mmol}, 0.81 \mathrm{~g})$ in acetic acid $(10 \mathrm{~mL})$ was refluxed for $12 \mathrm{~h}$. The mixture was concentrated under vacuum; water was added to the residue, filtered, dried, and crystallized from ethanol. Yield: $82 \%$; $\mathrm{mp} 202-203{ }^{\circ} \mathrm{C}$; IR $\left(\mathrm{KBr}, \mathrm{cm}^{-1}\right)$ : 3030 (CH aromatic), 2985, 2927 ( $\mathrm{CH}$ aliphatic), $1670(\mathrm{C}=\mathrm{O}) ;{ }^{1} \mathrm{H}-\mathrm{NMR}$ (DMSO-d $): \delta 3.90(\mathrm{~s}, 3 \mathrm{H}$, $\left.\mathrm{OCH}_{3}\right), 3.92\left(\mathrm{~s}, 3 \mathrm{H}, \mathrm{OCH}_{3}\right), 7.50-8.35(\mathrm{~m}, 9 \mathrm{H}, \mathrm{Ar}-\mathrm{H}) ;{ }^{13} \mathrm{C}-\mathrm{NMR}$ (DMSO): 52.3, 55.5, 103.5, $107.8,112.0,127.9,128.1,128.3,129.5,135.0,144.1,148.7,153.1,154.7,164.9,167.0,167.1$; MS, $m / z(\%): \mathrm{M}^{+}, 417$ (3.05\%), 55 (100\%). Anal. Calcd. for $\mathrm{C}_{21} \mathrm{H}_{15} \mathrm{~N}_{5} \mathrm{O}_{5}$ (417.37): C, 60.43; H, 3.62; N, 16.78. Found: C, 60.21; H, 3.71; N, 16.47\%.

\subsection{In Vitro Antitumor Activity Measurement against Human Mammary Carcinoma Cell Line (MCF7)}

Cells were plated in 96-multiwell plate (104 cells/well) for $24 \mathrm{~h}$ before treatment with the compounds to allow attachment of cell to the wall of the plate. Different concentrations of the compound under test $(0,1,2.5,5$ and $10 \mu \mathrm{g} / \mathrm{mL})$ were added to the cell monolayer. Triplicate wells were prepared for each individual dose. Monolayer cells were then incubated with the compounds for $48 \mathrm{~h}$ at $37{ }^{\circ} \mathrm{C}$ and in atmosphere of $5 \% \mathrm{CO}_{2}$. After this time, cells were fixed, washed, and stained with Sulforhodamine B stain. Excess stain was washed with acetic acid and attached stain was recovered with Tris EDTA buffer. The color intensity was measured in an ELISA reader. Finally, the relation between surviving fraction and drug concentration was plotted to get the survival curve of the tumor cell line after the specific compound [30]. The optical density measured is linear to the cell number of the surviving fraction. Therefore, the assay is a sensitive measure of compound induced cytotoxicity with the best signal to noise ratio. The assay also, provides a colorimeteric end point that is nondestructive, indefinitely stable and visible to naked eye. 


\subsection{D-QSAR Study}

\subsubsection{Data Set}

All the molecular modeling calculations and docking simulation studies were performed using Molecular Operating Environment $\left(\mathrm{MOE}^{\circledR}\right)$ version 10.2010, Chemical Computing Group Inc., Montreal, Canada. The computational software operated under Windows XP installed on an Intel Pentium IV PC with a $1.6 \mathrm{GHz}$ processor and $512 \mathrm{MB}$ memory. All the interaction energies and different calculations were automatically calculated. The biological activity values $\mathrm{IC}_{50}$ were converted to negative logarithmic $\left(\mathrm{P} \mathrm{IC}_{50}\right)$ and used as the dependant variable for the QSAR analysis. Thirteen different molecular descriptors (independent variables) were selected and calculated for the submitted structures aiming cover a wide range of different electronic, hydrophobic and topological characters. The correlation matrix was calculated to avoid multicolinearity between the calculated descriptors. The correlation matrix indicated that some of the descriptors used are highly correlated which suggests avoiding the combinations between such intercorrelated descriptors $(|r| \geq 0.80$, where $r$ is the simple linear coefficient). QSAR model was then constructed after ensuring reasonable correlation of antitumor activity with individual descriptor and minimum inter-correlation among the descriptors used in derived model.

\subsubsection{Statistical Analysis}

Stepwise linear regression analysis (SLRA) technique was used to test the best structural predictors for activity. For the current dataset of the new compounds, the QSAR model development was restricted to a maximum of three variables in accordance to the general accepted rule for the compounds: descriptors ratio to be around 5:1. The developed QSAR models are evaluated using the following statistical measures as root mean square error (RMSE) and correlation coefficient $\left(R^{2}\right)$ and validated by the leave-out technique (LOO technique), where each object of the data set is taken away, one at a time. In this case, given $\mathrm{n}$ objects, $\mathrm{n}$ reduced models are developed. The predicted activities $\left(p \mathrm{IC}_{50}\right)$ for the tested compounds calculated using multi-linear regression (\$Pred) technique.

\section{Conclusions}

Various substituted imidazo[1,5-a]quinazoline derivatives were synthesized. The structure of the newly synthesized compounds was elucidated by elemental analyses and spectral data. All compounds were tested, in vitro, for their antitumor activity. Compound 5a is the most active against the human mammary carcinoma cell line (MCF7). Physico-chemical parameters revealed that most of the compounds obeyed the "rule of five" properties with good absorption percentages. 2D-QSAR studies helpto explore some expectedly potent compounds.

\section{Acknowledgments}

Thanks are due to the National Cancer Institute, Cairo University for performing the antitumor testing of the synthesized compounds. Also, Author wishes to offer deep gratitude to Medicinal Chemistry Department, Faculty of Pharmacy, Assiut University for carrying the 2D-QSAR study. 


\section{Conflicts of Interest}

The authors declare no conflict of interest.

\section{References}

1. Siegel, R.; Naishadham, D.; Jemal, A. Cancer statistics, 2013. CA Cancer J. Clin. 2013, 63, $11-30$.

2. Hanahan, D.; Weinberg, R.A. The hallmarks of cancer. Cell 2000, 100, 57-70.

3. Vásquez, D.; Rodríguez, J.A.; Theoduloz, C.; Calderon, P.B.; Valderrama, J.A. Studies on quinones. Part 46. Synthesis and in vitro antitumor evaluation of aminopyrimidoisoquinolinequinones. Eur. J. Med. Chem. 2010, 45, 5234-5242.

4. Chen, X.; Du, Y.; Sun, H.; Wang, F.; Kong, L.; Sun, M. Synthesis and biological evaluation of novel tricyclic oxazine and oxazepine fused quinazolines. Part 1: Erlotinib analogs. Bioorg. Med. Chem. Lett. 2014, 24, 884-887.

5. Oue, N.; Anami, K.; Schetter, A.J.; Moehler, M.; Okayama, H.; Khan, M.A.; Bowman, E.D.; Mueller, A.; Schad, A.; Shimomura, M.; et al. High miR-21 expression from FFPE tissues is associated with poor survival and response to adjuvant chemotherapy in colon cancer. Int. J. Cancer 2014, 134, 1926-1934.

6. Yang, J.; Wang, L.J.; Liu, J.J.; Zhong, L.; Zheng, R.L.; Xu, Y.; Ji, P.; Zhang, C.H.; Wang W.J.; Lin, X.D.; et al. Structural optimization and structure-activity relationships of N2-(4-(4Methylpiperazin-1-yl)phenyl)-N8-phenyl-9H-purine-2,8-diamine derivatives, a new class of reversible kinase inhibitors targeting both EGFR-activating and resistance mutations. J. Med. Chem. 2012, 55, 10685-10699.

7. Cai, J.; Sun, M.; Wu, X.; Chen, J.; Wang, P.; Zong, X.; Ji, M. Design and synthesis of novel 4-benzothiazole amino quinazolines Dasatinib derivatives as potential anti-tumor agents. Eur. J. Med. Chem. 2013, 63, 7002-7012.

8. Sun, M.; Wu, X.; Chen, J.; Cai, J.; Cao, M.; Ji, M. Design, synthesis, and in vitro antitumor evaluation of novel diaryl ureas derivatives. Eur. J. Med. Chem. 2010, 45, 2299-2306.

9. Karpavicienea, I.; Cikotienea, I.; Padrón, J.M. Synthesis and antiproliferative activity of $\alpha$-branched $\alpha, \beta$-unsaturated ketones. Eur. J. Med. Chem. 2013, 70, 568-578.

10. Su, T.; Lee, T.; Kakadiya, R. The development of bis(hydroxymethyl)pyrrole analogs as bifunctional DNA cross-linking agents and their chemotherapeutic potential. Eur. J. Med. Chem. 2013, 69, 609-621.

11. Hussein, B.H.; Azab, H.A.; El-Azab, M.F.; El-Falouji, A.I. A novel anti-tumor agent, Ln(III) 2-thioacetate benzothiazole induces anti-angiogenic effect and cell death in cancer cell lines. Eur. J. Med. Chem. 2012, 51, 99-109.

12. Subtel'na, I.; Atamanyuk, D.; Szymanska, E.; Kieć-Kononowicz, K.; Zimenkovsky, B.; Vasylenko, O.; Gzella, A.; Lesyk, R. Synthesis of 5-arylidene-2-amino-4-azolones and evaluation of their anticancer activity. Bioorg. Med.Chem. 2010, 18, 5090-5102. 
13. Abdel Gawad, N.M.; Georgey, H.H.; Youssef, R.M.; El-Sayed, N.A. Synthesis and antitumor activity of some 2,3-disubstituted quinazolin-4(3H)-ones and 4, 6 disubstituted-1,2,3, 4-tetrahydroquinazolin-2H-ones. Eur. J. Med. Chem. 2010, 45, 6058-6067.

14. Al-Omary, F.A.M.; Abou-Zeid, L.A.; Nagi, M.N.; Habib, E.E.; Abdel-Aziz, A.A.M.; El-Azab, A.S.; Abdel-Hamide, S.G.; Al-Omar, M.A.; Al-Obaid, A.M.; El-Subbagh, H.I. Non-classical antifolates. Part 2: Synthesis, biological evaluation, and molecular modeling study of some new 2,6-substituted-quinazolin-4-ones. Bioorg. Med. Chem. 2010, 18, 2849-2863.

15. Al-Rashood, S.T.; Aboldahab, I.A.; Nagi, M.N.; Abouzeid, L.A.; Abdel-Aziz, A.A.M.; Abdel-Hamide, S.G.; Youssef, K.M.; Al-Obaid, A.M.; El-Subbagh, H.I. Synthesis, dihydrofolate reductase inhibition, antitumor testing, and molecular modeling study of some new 4(3H)-quinazolinone analogs. Biorg. Med. Chem. 2006, 14, 8608-8621.

16. Amin, K.M.; Georgey, H.H.; Awadallah, F.M. EGFR tyrosine kinase targeted compounds: Synthesis, docking study, and in vitro antitumor activity of some new quinazoline and benzo[d]isothiazole derivatives. Med. Chem. Res. 2011, 20, 1042-1053.

17. Rewcastle, G.W.; Palmer, B.D.; Bridges, A.J.; Showalter, H.D.; Sun, L.; Nelson, J.; McMichael, A.; Kraker, A.J.; Fry, D.W.; Denny, W.A. Tyrosine kinase inhibitors. 9. Synthesis and evaluation of fused tricyclic quinazoline analogues as ATP site inhibitors of the tyrosine kinase activity of the epidermal growth factor receptor. J. Med. Chem. 1996, 39, 918-928.

18. Wagner, G.; Bunk, E. Convalent binding of various tetra- and tricyclic heterocycles with isothiocyanate structure to human serum albumin and bovine gamma globulin. Part 24: Immunosuppressive agent-antigen conjugates. Pharmazie 1979, 34, 640-645.

19. Zhang, Y.; Chen, Z.; Lou, Y.; Yu, Y. 2,3-Disubstituted 8-arylamino-3H-imidazo[4,5-g]quinazolines: A novel class of antitumor agents. Eur. J. Med. Chem. 2009, 44, 448-452.

20. Abouzid, K.; Shouman, S. Design, synthesis and in vitro antitumor activity of 4-aminoquinoline and 4-aminoquinazoline derivatives targeting EGFR tyrosine kinase. Bioorg. Med. Chem. 2008, $16,7543-7551$.

21. Jin, Y.; Liu, J.; Huang, W.T.; Chen, S.W.; Hui, L. Synthesis and biological evaluation of derivatives of 4-deoxypodophyllotoxin as antitumor agents. Eur. J. Med. Chem. 2011, 46, 4056-4061.

22. Liu, X.H.; Li, J.; Shi, J.B.; Song, B.A.; Qi, X.B. Design and synthesis of novel 5-phenyl- $N$-piperidine ethanone containing 4,5-dihydropyrazole derivatives as potential antitumor agents. Eur. J. Med. Chem. 2012, 51, 294-299.

23. Mukhopadhyay, U.; Thurston, J.; Whitmire, K.H.; Siddi, Z.H.; Khokhara, A.R. Preparation, characterization, and antitumor activity of new cisplatin analogues with 1-methyl-4(methylamino)piperidine: Crystal structure of [PtII(1-methyl-4-(methylamino)piperidine) (oxalate)]. J. Inorg. Biochem. 2003, 94, 179-185.

24. Krezel, I. New derivatives of imidazole as potential anticancer agents. Farmaco 1998, 53, 342-345.

25. Kumar, R.; Gupta, L.; Pal, P.; Khan, S.; Singh, N.; Katiyar, S.B.; Meena, S.; Sarkar, J.; Sinha, S.; Kanaujiya, J.K.; et al. Synthesis and cytotoxicity evaluation of (tetrahydro-beta-carboline)-1,3,5triazine hybrids as anticancer agents. Eur. J. Med. Chem. 2010, 45, 2265-2276. 
26. El-Sherif, A.M.S.; Abdel-Maged, M.F.; Hammad, N.I.S.; Ammar, Y.A.; Harb, A.A. Imidazoles containing biologically active units: Synthesis of some 4-arylidene-2-aryl-5-oxo-4,5dihydroimidazoles. Egypt J. Chem. 1985, 28, 1-14.

27. Erlenmeyer, E.; Kreutz, A. Uber azlactone und ihre umwandlungsproducte. Annalen 1904, 337, 265-277.

28. Islam, A.M.; Khalil, A.M.; Abd El-Gawad, I.I. Reaction of 2-aryl-4-arylmethylene-2-oxazolin-5ones with amines. Aust. J. Chem. 1973, 26, 827-830.

29. Ragab, F.A.; Hassanein, H.H., Aly, E.I.; Georgy, H.H. Synthesis and pharmacological screening of certain imidazoquinazolone derivatives. Bull. Pharm. Sci. Assiut Univ. 2008, 31, 29-48.

30. Skehan, P.; Storeng, R.; Scudiero, D.; Monks, A.; McMahon, J.; Vistica, D.; Warren, J.R.; Bokesch, H.; Kenney, S.; Boyd, M.R. New colorimetric cytotoxicity assay for anticancer-drug screening. J. Natl. Cancer Inst. 1990, 82, 1107-1112.

31. Lipinski, C.A.; Lombardo, L.; Dominy, B.W.; Feeney, P.J. Experimental and computational approaches to estimate solubility and permeability in drug discovery and development settings. Adv. Drug Deliv. Rev. 2001, 46, 3-26.

32. Molinspiration Cheminformatics. Available online: http://www.molinspiration.com/services/ properties.html (accessed on 24 March 2014).

33. Zhao, Y.; Abraham, M.H.; Lee, J.; Hersey, A.; Luscombe, N.C.; Beck, G.; Sherborne, B.; Cooper, I. Rate-limited steps of human oral absorption and QSAR studies. Pharm. Res. 2002, 19, 1446-1457.

34. Hadjipavlou-Litina, D. Review, reevaluation, and new results in quantitative structure-activity studies of anticonvulsants. Med. Res. Rev. 1998, 18, 91-119.

Sample Availability: Samples of the newly synthesized compounds are available from the author.

(C) 2014 by the author; licensee MDPI, Basel, Switzerland. This article is an open access article distributed under the terms and conditions of the Creative Commons Attribution license (http://creativecommons.org/licenses/by/3.0/). 